\title{
Neuropathological and molecular comparison between clinical and asymptomatic bovine spongiform encephalopathy cases
}

\author{
Silvia Sisó • Marcus G. Doherr • Catherine Botteron • \\ Rosemarie Fatzer $\cdot$ Andreas Zurbriggen $\cdot$ \\ Marc Vandevelde • Torsten Seuberlich
}

Received: 7 June 2007 / Revised: 9 August 2007 / Accepted: 12 August 2007 / Published online: 1 September 2007

(C) Springer-Verlag 2007

\begin{abstract}
Interest in the proper neuropathological and molecular characterization of bovine spongiform encephalopathy (BSE) has increased since asymptomatic and atypical cases were detected in the cattle population by active disease surveillance. In this respect we investigated a total of 95 confirmed BSE cases originating from different active and passive surveillance categories (clinical suspects, emergency-slaughter, fallen stock and routinely slaughter) in Switzerland for their neuropathological and molecular phenotype. We looked for measurable differences between these categories in lesion profile, severity of spongiform change, degree of astrocytosis as well as immunohistochemical and molecular patterns of the disease-associated isoform of the prion protein $\left(\operatorname{PrP}^{\mathrm{d}}\right)$ in the caudal brainstem. Our results indicate significantly higher intensities of spongiform change in clinically affected compared to asymptomatic BSE cases. Similar effects were in trend observed for the intensities of $\operatorname{PrP}^{\mathrm{d}}$ deposition and astrocytosis, whereas the frequencies of morphological $\mathrm{PrP}^{\mathrm{d}}$ types and the molecular patterns in Western immunoblot were not different. Importantly, none of the animals included in this study revealed features of atypical BSE. Taken together, this study suggests that both clinically affected as
\end{abstract}

S. Sisó · M. G. Doherr · C. Botteron · R. Fatzer · A. Zurbriggen ·

M. Vandevelde $\cdot$ T. Seuberlich $(\square)$

NeuroCentre, Reference Laboratory for Transmissible

Spongiform Encephalopathies in Animals,

Department of Clinical Veterinary Medicine,

Vetsuisse Faculty, University of Berne,

Bremgartenstrasse 109A, 3001 Berne, Switzerland

e-mail: torsten.seuberlich@itn.unibe.ch

Present Address:

S. Sisó

VLA-Lasswade, Pentlands Science Park,

Bush Loan, Penicuik, Midlothian EH26 0PZ, UK well as asymptomatic Swiss BSE cases in cattle share the neuropathological and molecular phenotype of classical BSE and that asymptomatic classical BSE cases are at a pre-clinical stage of the disease rather than representing a true sub-clinical form of BSE.

Keywords Bovine spongiform encephalopathy · Prion . Atypical · Surveillance

\section{Introduction}

The epidemic nature of bovine spongiform encephalopathy (BSE) in the UK, its subsequent spread to most countries of Europe and the discovery of its zoonotic character in 1996 resulted in the continuous monitoring for BSE worldwide. Switzerland was the first country to complement passive disease surveillance by mandatory reporting of clinical suspects (CS), with a comprehensive active BSE surveillance program by targeting routinely slaughtered ("healthy") (RS) and emergency-slaughtered (ES) adult cattle as well as fallen stock (FS) [10]. This led to the first confirmation of BSE in cattle without any previous clinical BSE suspicion [24, 27]. Since 2001, similar BSE monitoring and epidemio-surveillance programmes were implemented in European Union member states and consistently led to an increase in detected BSE cases. As neurological signs were not reported in many of the confirmed cases, concerns were raised that sub-clinical forms of the disease may exist [20], viz. silent carriers as known from other important infectious animal diseases $[23,30]$, that in contrast to animals in a preclinical stage would not reveal clinical signs during their normal live span.

More recently, atypical variants of BSE have been identified by active surveillance programmes first in Italy [7], 
France [3] and Japan [34], and later in a series of other countries $[1,4]$. All these cases showed an aberrant molecular phenotype of the proteinase $\mathrm{K}$ resistant part of the pathological prion protein $\left(\mathrm{PrP}^{\mathrm{res}}\right)$ in Western immunoblot (WB) analysis and a noticeable old age when compared to classical BSE affected cattle. With the exception of one case in a zebu [28], all such atypical BSE cases were identified by active surveillance and therefore clinical features were not reported or remained ambiguous [4], further supporting the notion that sub-clinical BSE forms may indeed exist.

The characterization of the phenotype of transmissible spongiform encephalopathies (TSE) includes lesion profiles in the central nervous system (CNS) [29], molecular typing of $\mathrm{PrP}^{\text {res }}$ by WB [21] and transmission studies to animal models [5]. Recently, the neuroanatomical distribution and morphological type of the disease-associated isoform of the prion protein $\left(\mathrm{PrP}^{\mathrm{d}}\right)$ as determined by Immunohistochemistry (IHC) in the obex region of the caudal brainstem were established as additional criteria to distinguish between BSE phenotypes [8].

Since the Swiss BSE epidemic was monitored by both, passive and active surveillance, we had the opportunity to collect brain material from bovines with typical BSE neurological signs as well as confirmed cases of BSE detected from any of the active surveillance streams (ES, FS, RS). Categories ES and FS include cases without BSE-associated neurological signs, and RS cattle did not have any clinical history. All animals were diagnosed between 1999 and 2002 with an equal spread over time of the categories and the presence of $\mathrm{PrP}^{\mathrm{d}}$ in the CNS was confirmed by IHC on obex sections. We looked for measurable neuropathological differences in the caudal brainstem between animals of these categories according to the lesion profile, severity of spongiform change, degree of astrocytosis as well as immunohistochemical and molecular $\operatorname{PrP}^{\mathrm{d}}$ patterns. The purpose of this study was to answer the question whether asymptomatic BSE cases differ in these phenotypic features from clinical BSE cases, to assess whether they could be classified as sub-clinical or rather pre-clinical BSE and if any of those cases show patterns indicative of atypical BSE.

\section{Materials and methods}

Tissue samples

Between January 1999 and August 2002 a total of 148 domestic bovine BSE cases had been submitted to our laboratory for confirmatory diagnosis. Based on the availability of tissues and a preliminary assessment of the tissue quality in terms of autolysis, 95 brainstem samples were found to be appropriate for a neuropathological assessment and were included in the present study. Five non-BSE brainstems served as negative control samples in order to confirm the specificity of the techniques and antibodies used. Samples were grouped into four different categories based on the surveillance stream that they originated from (a) clinical BSE suspected animals representing cows with neurological signs suggestive of BSE, (b) ES cattle which were removed from the population for health-related reasons and were slaughtered sick, (c) FS which were found dead or culled due to health or age related problems and having their carcass subsequently disposed off and (d) RS cattle, which were considered to be slaughtered healthy (Table 1). From all cases, the half brainstem was fixed in $4 \%$ formaldehyde for 7-15 days, treated in $96 \%$ formic acid for $1 \mathrm{~h}$ and embedded in paraffin for further routine histopathological and immunohistochemical examination. The second half was frozen and used for BSE screening tests and molecular $\mathrm{PrP}^{\mathrm{res}}$ typing.

\section{Immunohistochemistry}

For IHC, 4- $\mu \mathrm{m}$ sections of the obex were cut and immunolabelled for the presence of the $\operatorname{PrP}^{\mathrm{d}}$ and glial fibrillary acidic protein (GFAP). For $\operatorname{PrP}^{\mathrm{d}}$ detection, slides were subjected to hydrated autoclaving at $121^{\circ} \mathrm{C}, 1 \mathrm{~b}$, for $30 \mathrm{~min}$ in distilled water, then treated with $98 \%$ formic acid (Merck) for $5 \mathrm{~min}$ at room temperature and finally treated with $5 \mu \mathrm{g} /$ $\mathrm{ml}$ proteinase $\mathrm{K}$ (Roche) for $15 \mathrm{~min}$ at $37^{\circ} \mathrm{C}$. Endogenous peroxidase activity was inhibited with $0.03 \%$ hydrogen peroxide (Merck) for $15 \mathrm{~min}$, followed by washing in distilled water. Then, slides were incubated overnight at $4^{\circ} \mathrm{C}$ either with the rabbit-polyclonal antibody $\mathrm{C} 15 \mathrm{~S}$ [17] at dilution of 1:800 in PBS or the rabbit-polyclonal anti-GFAP (Dako, no pre-treatment, 1:600). After washing, sections were processed with the Chemate ${ }^{\mathrm{TM}}$ Detection Kit Peroxidase/AEC Rabbit/Mouse (Dako) following the instructions of the

Table 1 Numbers of examined animals and confirmed BSE cattle cases in the frame of active and passive surveillance in Switzerland from 1999 to 2002

\begin{tabular}{lccl}
\hline Surveillance category & \multicolumn{2}{l}{ Numbers of animals } \\
\cline { 2 - 4 } & Examined & $\begin{array}{l}\text { Confirmed } \\
\text { BSE }\end{array}$ & $\begin{array}{l}\text { Included in the } \\
\text { present study }\end{array}$ \\
\hline Passive surveillance & & & \\
Clinical suspect (CS) & 451 & 59 & 45 \\
Active surveillance & & & \\
Emergency & 24,327 & 28 & 18 \\
$\quad$ slaughter (ES) & 33,023 & 38 & 18 \\
Fallen stock (FS) & 335,119 & 23 & 14 \\
Regular slaughter (RS) & 392,920 & 148 & 95 \\
Total & &
\end{tabular}


supplier. Slides were weakly counterstained with aqueous haematoxylin before mounting (Glycergel, Dako).

\section{Assessment of BSE-associated pathology}

Spongiform change was semi-quantitively assessed in five different neuroanatomical areas in the obex region: the dorsal motor nucleus of the vagus nerve (DMNV), the solitary tract nucleus (ST), the reticular formation (RF), the spinal tract nucleus of the trigeminal nerve (STT) and the inferior olives nuclei (ON). For practical purposes, the DMNV and ST were grouped together and scored as single nucleus (DMNV-ST). Two trained pathologists scored all slides independently and blindly. First analyses showed that scores did not differ significantly between the two investigators (non-parametric test for paired observations). Therefore, the scores assigned by one investigator were used for further analysis. The lesion scoring was done from 0 to 5 following the criteria described in mouse models of scrapie [15] rather than that adapted for cattle with BSE with ranges from 0 to 4 following the criteria described previously for cattle with BSE from 0 to 4 [32]: 0 , no vacuoles; 1 , few vacuoles; 2 , few vacuoles evenly scattered; 3 , moderate numbers of vacuoles; 4, many vacuoles with some confluence and 5, dense vacuolation with most of field confluent.

Disease-associated prion protein deposition was semiquantitively scored from 0 , no $\operatorname{PrP}^{\mathrm{d}}$ to 5 , severe $\operatorname{PrP}^{\mathrm{d}}$ deposition in the same neuroanatomical structures as per the lesion scores. Moreover, the presence of different morphological $\operatorname{PrP}^{\mathrm{d}}$ types was determined in each of the examined nuclei as fine punctate granular, coarse granular, coalescing, glial (stellate), linear, perineuronal, intraneuronal or intraglial.

Finally, GFAP-positive astrocytes were overall semiquantitively scored for obex sections: 1 , mild; 2 , moderate; 3 , severe astrocytosis.

\section{Western immunoblotting}

Tissue samples of the caudal brainstem, in some cases of the thalamus, were analysed in duplicate by WB following the protocol of the commercial Prionics Check Western Kit (Prionics) using the PrP specific monoclonal antibodies (mAb) 6H4 (Prionics) and P4 (R-biopharm) separately with the following modifications: ten well $12 \%$ NuPage gels (Invitrogen) were used, the secondary antibody was a rabbit-anti-mouse-HRP-conjugate (Dako, 1:3,000) and the detection was done with the ECL plus kit (Amersham Biosciences). For each gel the two outer lanes were loaded with the precision ${ }^{\circledR}$ all-blue molecular mass marker (Bio-Rad). For the molecular PrP $^{\text {res }}$ characterization, photographic films were scanned and the molecular masses and relative intensities of the diglycosylated, monoglycosylated and unglycosylated $\mathrm{PrP}^{\text {res }}$ moieties were measured for each sample using the Quantity-One Software (Bio-Rad).

\section{Statistical analyses}

Lesion scores and $\operatorname{PrP}^{\mathrm{d}}$ scores were summarized using medians and ranges, and visualized by box plots. For comparison of these scores as well as animal age between the four surveillance streams, a Kruskal-Wallis ANOVA on Ranks with post hoc Kruskal-Wallis multiple comparison $Z$-test and Bonferroni correction for multiple comparison was employed. Correlation between the lesion- and $\operatorname{PrP}^{\mathrm{d}}$ scores as well as between different anatomical regions were assessed by the Spearman Rank correlation coefficient (rSp). All statistics were done with NCSS 2004 (www.ncss.com), and the alpha level for statistical significance was set to 0.05 .

\section{Results}

Spongiform lesions and $\operatorname{PrP}^{\mathrm{d}}$ deposition scores

Spongiform change was assessed in four different neuroanatomical structures of the obex region (Fig. 1, lesion score). For all animal categories the median of the lesion score was highest either in the DMNV-ST or the STT and lowest in the ON and RF. In CS animals the DMNV-ST and the STT displayed the highest median of the lesion scores (3.0) compared to the lowest median scores found in the RF (1.0). For ES and FS but not for RS cattle, the STT revealed lesion scores as high as for CS cattle (median 3.0). By contrast in the DMNV-ST and the ON spongiform lesions for ES and FS tended to score lower compared to CS and rather similar to RS animals. rSp of $0.47-0.50$ indicated low correlations of lesion scores between the structures under investigation.

Regarding the intensities of $\operatorname{PrP}^{\mathrm{d}}$ deposition (Fig. 1, bottom panel) the highest median scores were found in the DMNV-ST of CS (3.0) and ES (3.0) animals. The median $\mathrm{PrP}^{\mathrm{d}}$ scores of RS cases consistently scored lower compared to those in CS animals, although not statistically significantly. Similar to the spongiform lesion scores, $\mathrm{PrP}^{\mathrm{d}}$ scores in ES and FS animals were close to those of CS animals in the STT, but in the ON resembled those of RS animals. Analyses for the correlation of $\mathrm{PrP}^{\mathrm{d}}$ scores between these structures revealed rSps ranging from 0.54 to 0.68 , indicating a moderate correlation.

Overall, the correlation between $\operatorname{PrP}^{\mathrm{d}}$ deposition and spongiform lesions in the respective nuclei was at best low (rSps 0.3-0.51). 

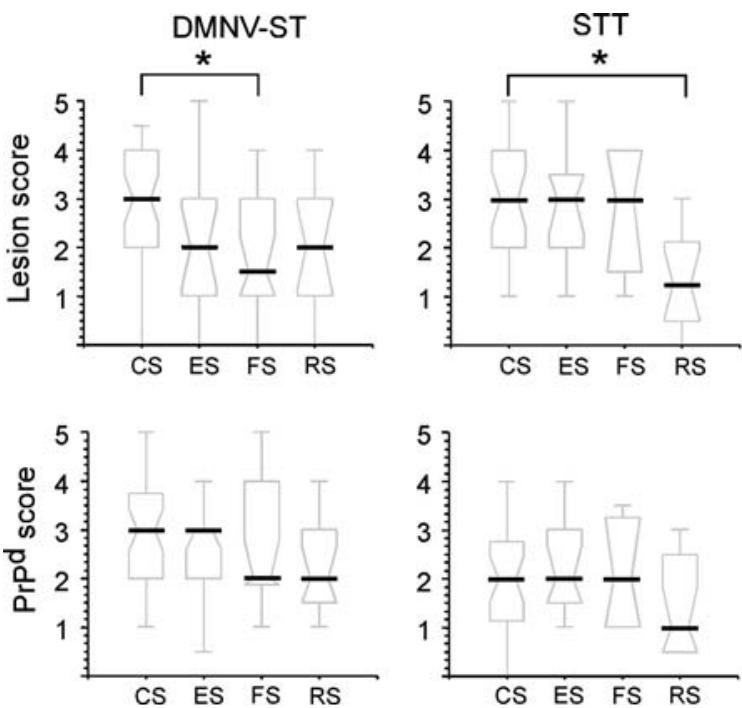

Fig. 1 Spongiform change- and $\operatorname{PrP}^{\mathrm{d}}$ scoring for clinical suspect $(C S)$, emergency-slaughtered $(E S)$, fallen stock $(F S)$ and routinely slaughtered $(R S)$ BSE confirmed cattle in four neuro-anatomical structures of the caudal brainstem (obex level). Boxes represent the 25th, 50th (median: narrow section with bold line) and 75th percentile of the score distribution within groups. Whiskers approximate the central $95 \%$ of the data. Outliers are shown as dots. Statistical analysis resulted in

\section{Morphological $\operatorname{PrP}^{\mathrm{d}}$ deposition types}

Eight different $\operatorname{PrP}^{\mathrm{d}}$ types were identified in the obex region by IHC using the rabbit-polyclonal anti-PrP antibody C15S. Noteworthy, none of the BSE cases included in this study showed $\operatorname{PrP}^{\mathrm{d}}$ - immunopositive amyloidotic plaques. In the DMNV-ST and the STT the fine punctuate and the coarse granular morphological $\operatorname{PrP}^{\mathrm{d}}$ types were the most predominant (Fig. 2). In contrast to the rest of the nuclei, the intraneuronal $\operatorname{PrP}^{\mathrm{d}}$ immunolabelling was common in the ON whereas the stellate type completely absent. Distinctively, the linear $\operatorname{PrP}^{\mathrm{d}}$ type predominated in the RF.

When we compared the four animal categories the frequency of $\mathrm{PrP}^{\mathrm{d}}$ deposition types in the each neuroanatomical structure was remarkably constant (Fig. 2). However, a striking difference was that for the coalescing $\operatorname{PrP}^{\mathrm{d}}$ type the frequencies overall dropped down from CS and ES to FS animals and that this type was not detected in any of the examined nuclei in RS cattle.

\section{Astrocytosis}

The median scores of the overall gliosis in the obex tissue sections were 2.5, 2, 1.75 and 1.5 for CS, ES, FS and RS animals, respectively. However, despite this clear trend, differences in the scores between the four animal categories were not statistically significant due to rather broad variations (data not shown).

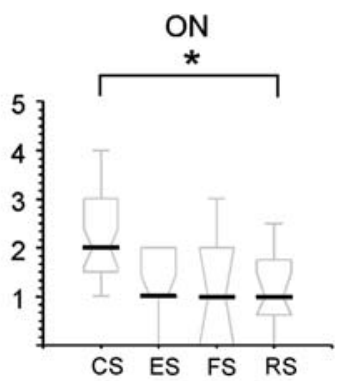

RF
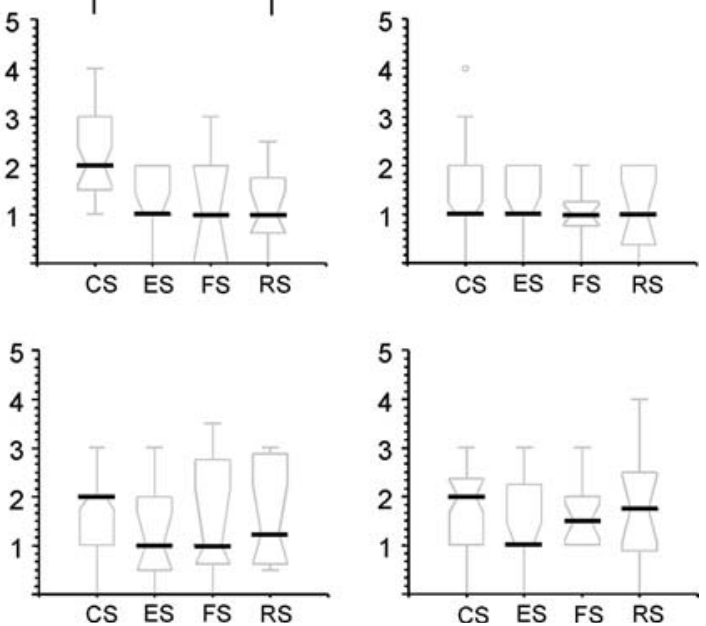

significant differences $(P<0.05$, indicated by asterisks $)$ in the lesion scores (spongiform change) in the dorsal motor nucleus of the vagus nerve/solitary tract nucleus $(D M N V-S T)$, the olivary nuclei $(O N)$ and the spinal tract nucleus of the trigeminal nerve (STT) but not in the reticular formation $(R F)$ between categories. By contrast, for $\operatorname{PrP}^{\mathrm{d}}$ scoring although clear trends in median scores between categories are identifiable, differences are not statistically significant

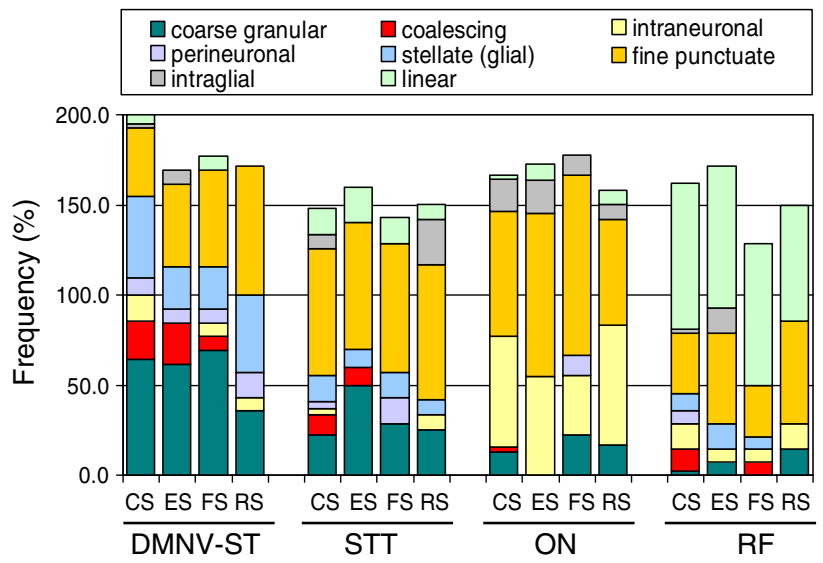

Fig. 2 Frequencies of morphological $\operatorname{PrP}^{\mathrm{d}}$ deposition types in the four brain stem nuclei under investigation (obex region) in BSE confirmed cattle. Note that each nucleus revealed a variety of types which differ in proportion and that multiple types per nucleus were frequently observed. Differences were identified not only when nuclei were compared but also between animals that derived from the four BSE surveillance stream categories (see text)

Molecular characterization

For a total of 71 BSE confirmed cases appropriate frozen CNS tissues were available. All cases showed a typical three-band pattern consisting of two PrP $^{\text {res }}$ glycoforms of approximately 22 and $27 \mathrm{kDa}$, and a unglycosylated moiety of approximately $18 \mathrm{kDa}$ in molecular mass by WB analysis using $\mathrm{mAb} 6 \mathrm{H} 4$. The mean values of the molecular 
masses of the animals of the four categories were essentially undistinguishable (Fig. 3a). Variations, especially of the molecular mass of the unglycosylated peptide were only to a minor extent within and between the four categories. Also by visual side-by-side comparison no obvious differences in the molecular masses of $\mathrm{PrP}^{\mathrm{res}}$ in individual samples were observed. Similarly, the proportions of the three $\mathrm{PrP}^{\mathrm{res}}$ moieties did not reveal any obvious differences between the categories although some variation was found. Importantly, the diglycosylated moiety was predominant in all samples under investigation (Fig. 3b) and exceeded 50\% of the total $\mathrm{PrP}^{\mathrm{res}}$ signal intensity in each case. None of the samples, except for non-proteinase $\mathrm{K}$ treated controls reacted with $\mathrm{mAb} \mathrm{P} 4$.

\section{Discussion}

In this retrospective study we present a systematic neuropathological and molecular comparison of BSE cases from 1999 to 2002 in Switzerland. The identification of BSE affected cattle by passive surveillance relies on the reporting of animals with clinical signs indicative for BSE (CS),
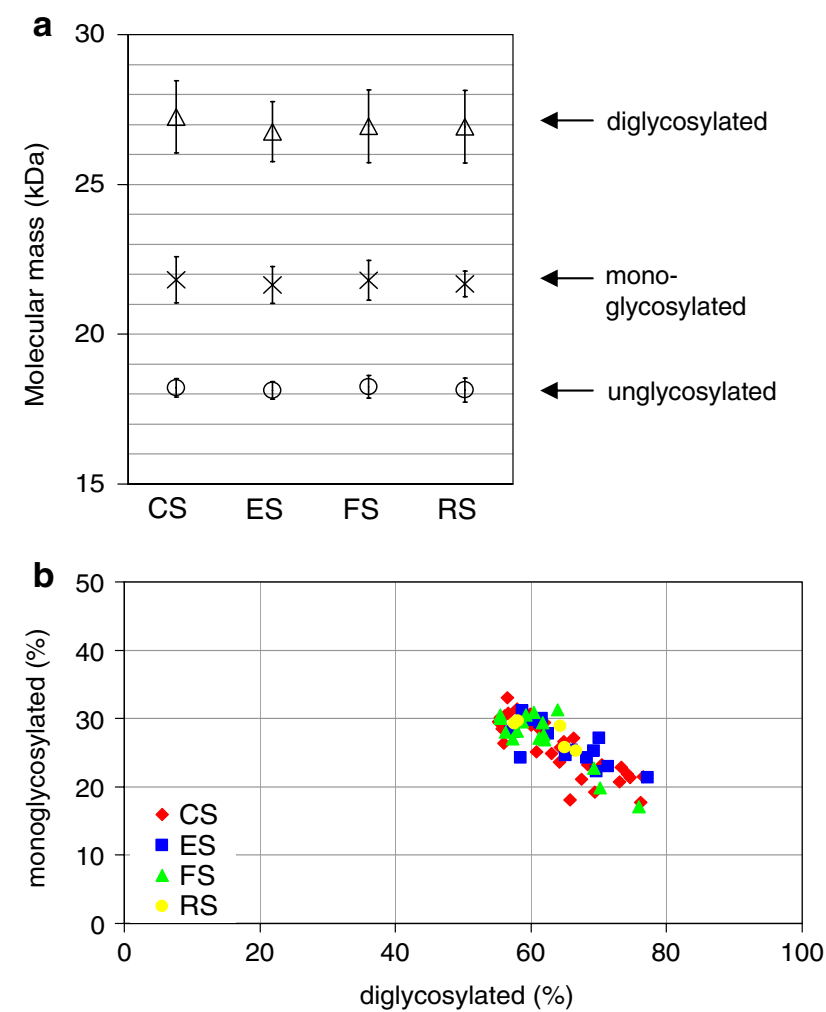

Fig. 3 Molecular typing of $\operatorname{PrP}^{\text {res }}$ by Western immunoblotting. Molecular masses (a) and relative proportions (b) of the mono- and diglycosylated $\mathrm{PrP}^{\mathrm{res}}$ moieties in BSE confirmed cattle. CS clinical suspect $(n=33), E S$ emergency slaughter $(n=16), F S$ fallen stock $(n=17), R S$ routine slaughter $(n=5)$ followed by post mortem laboratory confirmation of the disease by one of the confirmatory methods listed by the Office International de Epizooties [25]. Thus, CS BSE cases can per se be regarded as clinically BSE affected. For active surveillance of FS cattle, direct neurological clinical data are usually not collected. These animals were either in an asymptomatic BSE state or died/were culled for other reasons and clinical BSE signs remained unrecognised or were not reported. The situation for RS and ES cattle is different in so far as according to Swiss regulations cattle intended for slaughter undergo ante mortem inspection complemented by BSE-specific clinical examination procedures for those over 30 month of age [19]. RS cattle passed this barrier and therefore are likely clinically healthy. However, for ES cattle BSE unrelated symptoms might have masked specific neurological signs and consequently such cases had not been recognized as CS. Taking these assumptions together we expect that animals identified by passive surveillance were clinically BSE affected and that the vast majority of RS cases were indeed asymptomatic for BSE. FS and ES cases were either of both and it is difficult to assess to which extend clinical cases were missed in these categories. It was estimated that the odds ratio to detect BSE in ES and FS animals was $~ 50$ compared to RS animals [10] and it is adequate to postulate, that a proportion of them was clinically affected, while others were healthy.

All CS, ES and FS BSE cases showed spongiform lesions in the STT and, with the exception of one animal for each category, also in the DMNV-ST. For the RS category two animals lacked spongiform lesions in the STT but not in the DMNV-ST. These findings are in accordance with previous studies on lesion profiles in BSE done in the UK [29, 31, 33], Switzerland [14, 17] and elsewhere [9, 26]. All agreed on the identification of those three obex nuclei as primary target structures for routine histopathological BSE diagnostics. Taken additionally into account that we found a similar obex topography of $\operatorname{PrP}^{\mathrm{d}}$ types (Fig. 2) as that found in Italian and British classical BSE cattle [8], our data strengthen former studies on clinically BSE affected Swiss cattle that revealed a histopathological BSE phenotype similar to British BSE cases [17, 18] and extend this conclusion to asymptomatic BSE cattle identified in frame of active surveillance in Switzerland.

The highest lesion scores for spongiform change in all studied nuclei corresponded to CS and the lowest to RS animals. These differences were statistically significant in the STT and the ON. Likewise $\operatorname{PrP}^{\mathrm{d}}$ scores tended to be higher in CS as compared to RS animals. Depending on the nuclei, ES and FS BSE cases overall scored more or less intermediate in both criteria. We also found the highest frequencies of coalescing $\operatorname{PrP}^{\mathrm{d}}$ in $\mathrm{CS}$, while it was absent in RS BSE cases. Moreover astrocytosis was in trend increasing from RS over FS and ES to the CS category. 
In sum these findings indicate that spongiform lesions, $\operatorname{PrP}^{\mathrm{d}}$ deposits as well as astrogliosis and $\operatorname{PrP}^{\mathrm{d}}$ aggregation in the obex region are overall more severe in clinically affected as compared to asymptomatic BSE cases. While $\operatorname{PrP}^{\mathrm{d}}$ deposition by itself does not imply clinical disease, it is not inappropriate to speculate that due to the slow progression of the disease and the long incubation period which characterizes TSE infections, these findings reflect a progression of the disease. Together with the uniform histopathological and immunohistochemical phenotype this implicates that asymptomatic cattle are rather in a preclinical state and unlikely remain per se sub-clinical. If so, one would expect that animals that displayed BSE clinical signs were older than those belonging to the RS category, and similarly that animals with the highest lesion scores were the oldest because of a longer incubation period. The median age at death was 64.5 months in CS, ES and FS and 74.5 months in RS cattle. Although the age range was rather wide (43-142 months, with five animals older than 100 month) we were not able to establish a statistical correlation between the age of the animals, the clinical status and the lesion severity (data not shown). This lack of correlation may be due to rather small numbers of animals especially in the RS category or other uncertainties that are still related to the pathogenesis of BSE especially under field conditions, like differences in time points of infection, individual susceptibilities, infectious doses or other factors. A limitation of our study is the restriction to brainstem/obex tissues. Differences between cases and categories may have been much more obvious when other parts of the brain, which may become involved at later stages in the disease, were included in the assessment. However, we were limited to the brainstem in terms of consistency, because of sampling being limited to the caudal brainstem in a large number of animals. Therefore, we agree that an experimental BSE cattle model in which all those variables are under control and complete brain is available would be required to provide additional and more conclusive evidence for the absence or the putative phenotype of sub-clinical BSE infections.

In one point our results disagree with those described by Casalone et al. [8]. Unexpectedly and contrary to our results, they found a greater intensity of $\operatorname{PrP}^{\mathrm{d}}$ immunolabelling in the active surveillance animal population (considered as healthy) compared to that recorded in clinical cases. They speculated that active surveillance may "select" for animals that show a higher degree of $\operatorname{PrP}^{\mathrm{d}}$ deposition prior to onset of clinical disease compared to others. A possible explanation for such an effect may be differences in the diagnostic sensitivity of the respective screening tests compared to that of the confirmatory procedures. In case that a screening tests applied in active surveillance is less sensitive than the confirmatory test, it may select for cases with stronger, while the latter also identifies cases with weaker $\operatorname{PrP}^{\mathrm{d}}$ deposits when it is applied to CS animals. Indeed, to our knowledge, none of the evaluation studies of BSE screening tests carried out so far [11-13] included positive samples derived from active surveillance schemes, but were based on the examination of clinically BSE affected animals exclusively. Thus, the performance of the screening test on asymptomatic BSE cases especially also in comparison with established protocols for confirmatory diagnosis is still undetermined. Another factor that might contribute to such a "selection" is the common difficulty to identify and sample the appropriate target area in active surveillance samples due to tissue autolysis (esp. for FS) or the inappropriate use of sampling devices thereby missing the target nuclei in the brainstem whereas for instance in IHC these structures can be identified visually. In addition, to our experience the use of sampling syringes provided by some screening tests which have been widely used since 2001 in the European Union, clearly results in an increased risk of missing the diagnostic target areas in the caudal brainstem whereas the Prionics Check Western test [27], which was used for the vast majority of active BSE surveillance in Switzerland between 1999 and 2004, uses hemi-sections of the obex region that appear to contain these structures more likely. Different sampling and screening test procedures for active surveillance in Switzerland therefore possibly compensate for such "selection" effects.

Atypical BSE is currently classified as H-type (high) or L-type (low) according to the apparent molecular mass of the unglycosylated $\mathrm{PrP}^{\mathrm{res}}$ fragment when compared to classical BSE by WB analysis using mAbs that bind to the central part of $\mathrm{PrP}^{\mathrm{res}}$, like 6H4 [21]. Such differences are in the range of $1.4 \mathrm{kDa}$ for H-type but very subtle or even absent for the L-type $[6,21]$. By contrast the latter reveals a less intense band of the diglycosylated $\mathrm{PrP}^{\text {res }}$ isoform $\left(<50 \%\right.$ of the total $\left.\operatorname{PrP}^{\mathrm{res}}\right)$ whereas in classical BSE it was found to be more prominant ( $>50 \%$ ) [21]. Additionally, in $\mathrm{H}$-type cases mAbs binding to the $\mathrm{N}$-terminus (e.g. P4) readily detect $\operatorname{PrP}^{\text {res }}$, which is not the case for classical and L-type BSE. Of all cases investigated in the present study by WB with mAbs $6 \mathrm{H} 4$ and $\mathrm{P} 4$ none revealed molecular features of H-type or L-type BSE and no differences in the molecular phenotype between the four categories were identified. It must be emphasized that for 24 animals appropriate frozen tissues were not available. Consequently the molecular phenotype for these animals could not be determined. For the remaining 71 cases it was clearly that of classical BSE.

Little is known about the histopathological and immunohistochemical characteristics of atypical BSE. In two Italian cases, Casalone et al. [7, 8] described the presence of $\operatorname{PrP}^{\mathrm{d}}$ positive amyloidotic plaques mainly in the RF and the nucleus of the STT of the brainstem as pathognomonic for 
L-type BSE, thus also termed bovine amyloidotic spongiform encephalopathy (BASE). These animals revealed a neuroanatomical $\operatorname{PrP}^{\mathrm{d}}$ distribution distinct from classical BSE and involved mainly the more rostral parts of the brain and to a lesser extent the caudal brainstem. By contrast amyloidotic plaques have not yet been described in bovine $\mathrm{H}$-type BSE cases, but in the brain of mice after transmission of the H-type agent [2]. Recently we reported a miniature zebu presenting an $\mathrm{H}$-type molecular $\mathrm{PrP}^{\mathrm{res}}$ pattern [28]. The type and distribution of the histopathological lesions as well as the $\operatorname{PrP}^{\mathrm{d}}$ depositions in the brain were undistinguishable from classical BSE in cattle. Whether this also applies for H-type BSE in cattle still remains to be determined. With the current knowledge and low incidence it appears difficult to discriminate H-type BSE cases on the basis of the PrP deposition types from classical BSE. However, it is possible that suitable tests based on the same principle as the epitope mapping IHC used for TSE strain discrimination in small ruminants $[16,22]$ could be developed in the near future. None of the animals investigated here, including the 24 cases that remained unclassified so far, revealed plaque- like $\operatorname{Pr}^{\mathrm{d}}$ depositions indicative for L-type BSE (or BASE).

In conclusion, the present retrospective study shows that the neuropathological phenotype of the Swiss BSE cases included in this study and the molecular phenotype of all cases where appropriate tissue was available is that of classical BSE and remains remarkably constant irrespective of the surveillance stream and therefore the clinical BSE status. However, significant differences in BSE related pathology exist between clinical and asymptomatic BSE cases, but are related to severity and spread of spongiform lesions in brainstem nuclei and can be explained by the progressive nature of the disease. Thus, these results suggest that asymptomatic animals with confirmed BSE were at a preclinical stage of the disease rather than representing a true sub-clinical form of BSE. Therefore, this study does not support the notion that classical BSE would be maintained as a clinically silent form of BSE in the cattle population. None of the BSE animals under investigation showed features of atypical BSE, but this study was limited in sample size and if atypical BSE as often speculated represents a sporadic TSE in bovine that mainly affects older animals, than its prevalence in a given population should be directly proportional to the numbers of older animals tested. Although the Swiss H-type zebu revealed clinical neurological signs indicative for BSE, we do not know whether this also applies for H- and L-type BSE cattle and consequently they may not fall into the CS surveillance category, which was highly represented, in the present study. Considering these caveats, ongoing more extended work will clarify whether and to which extend atypical BSE cases are part of the Swiss BSE epidemic.
Acknowledgements The authors acknowledge Nathalie Ligeti, Valérie Juillerat and Doris Ambühl for excellent technical assistance. We would like to thank Dr Lorenzo González and Dr Martin Jeffrey for critical reading of the manuscript and helpful discussions as well as Heinzpeter Schwermer for providing BSE surveillance data. The Swiss Federal Veterinary Office funded this study.

\section{References}

1. Baron T, Biacabe AG, Arsac JN, Benestad S, Groschup MH (2006) Atypical transmissible spongiform encephalopathies (TSEs) in ruminants. Vaccine 25:5625-5630

2. Baron TG, Biacabe AG, Bencsik A, Langeveld JP (2006) Transmission of new bovine prion to mice. Emerg Infect Dis 12:11251128

3. Biacabe AG, Laplanche JL, Ryder S, Baron T (2004) Distinct molecular phenotypes in bovine prion diseases. EMBO Rep 5:110-115

4. Brown P, McShane LM, Zanusso G, Detwiler L (2006) On the question of sporadic or atypical bovine spongiform encephalopathy and Creutzfeldt-Jakob disease. Emerg Infect Dis 12:18191821

5. Bruce ME, Will RG, Ironside JW, McConnell I, Drummond D, Suttie A, McCardle L, Chree A, Hope J, Birkett C, Cousens S, Fraser H, Bostock CJ (1997) Transmissions to mice indicate that 'new variant' CJD is caused by the BSE agent. Nature 389:498-501

6. Buschmann A, Gretzschel A, Biacabe AG, Schiebel K, Corona C, Hoffmann C, Eiden M, Baron T, Casalone C, Groschup MH (2006) Atypical BSE in Germany-proof of transmissibility and biochemical characterization. Vet Microbiol 117:103-116

7. Casalone C, Zanusso G, Acutis P, Ferrari S, Capucci L, Tagliavini F, Monaco S, Caramelli M (2004) Identification of a second bovine amyloidotic spongiform encephalopathy: molecular similarities with sporadic Creutzfeldt-Jakob disease. Proc Natl Acad Sci U S A 101:3065-3070

8. Casalone C, Caramelli M, Crescio MI, Spencer YI, Simmons MM (2006) BSE immunohistochemical patterns in the brainstem: a comparison between UK and Italian cases. Acta Neuropathol (Berl) 111:444-449

9. Debeer S, Baron T, Bencsik A (2003) Neuropathological characterisation of French bovine spongiform encephalopathy cases. Histochem Cell Biol 120:513-521

10. Doherr MG, Heim D, Fatzer R, Cohen CH, Vandevelde M, Zurbriggen A (2001) Targeted screening of high-risk cattle populations for BSE to augment mandatory reporting of clinical suspects. Prev Vet Med 51:3-16

11. EFSA (2004) Scientific report on the evaluation of seven new rapid post mortem BSE tests. http://www.efsa.europa.eu/etc/medialib/efsa/ science/tse_assessments/bse_tse/694.Par.0002.File.dat/sreport18_ 7new_rpmt_bse_en1.pdf

12. European Commission (1999) The evaluation of tests for the diagnosis of transmissible spongiform encephalopathy in bovines. http://www.europa.eu.int/comm/food/fs/bse/bse12_en.pdf

13. European Commission (2002) The evaluation of five rapid tests for the diagnosis of transmissible spongiform encephalopathy in bovines (2nd study). http://www.europa.eu.int/comm/food/fs/bse/ bse42_en.pdf

14. Fatzer R, Graber HU, Meyer RK, Cardozo C, Vandevelde M, Zurbriggen A (1996) Neuronal degeneration in brain stem nuclei in bovine spongiform encephalopathy. Zentralbl Veterinarmed A 43:23-29

15. Fraser H, Dickinson AG (1968) The sequential development of the brain lesion of scrapie in three strains of mice. J Comp Pathol 78:301-311 
16. Gonzalez L, Martin S, Houston FE, Hunter N, Reid HW, Bellworthy SJ, Jeffrey M (2005) Phenotype of disease-associated PrP accumulation in the brain of bovine spongiform encephalopathy experimentally infected sheep. J Gen Virol 86:827-838

17. Graber HU, Meyer RK, Fatzer R, Vandevelde M, Zurbriggen A (1995) In situ hybridization and immunohistochemistry for prion protein $(\mathrm{PrP})$ in bovine spongiform encephalopathy (BSE). Zentralbl Veterinarmed A 42:453-459

18. Gubler E, Hilbe M, Ehrensperger F (2007) Lesion profiles and gliosis in the brainstem of 135 Swiss cows with bovine spongiform encephalopathy (BSE). Schweiz Arch Tierheilkd 149:111-122

19. Hett AR, Rufenacht J, Perler L, Heim D (2002) [A method to identify BSE suspects in emergency slaughtered cattle during ante mortem examination]. Schweiz Arch Tierheilkd 144:654-662

20. Hill AF, Collinge J (2003) Subclinical prion infection. Trends Microbiol 11:578-584

21. Jacobs JG, Langeveld JP, Biacabe AG, Acutis PL, Polak MP, Gavier-Widen D, Buschmann A, Caramelli M, Casalone C, Mazza M, Groschup M, Erkens JH, Davidse A, Van Zijderveld FG, Baron $\mathrm{T}$ (2007) Molecular discrimination of atypical bovine spongiform encephalopathies from a wide geographical region in Europe. J Clin Microbiol 45:1821-1829

22. Jeffrey M, Martin S, Gonzalez L, Foster J, Langeveld JP, Van Zijderveld FG, Grassi J, Hunter N (2006) Immunohistochemical features of $\operatorname{PrP}(\mathrm{d})$ accumulation in natural and experimental goat transmissible spongiform encephalopathies. J Comp Pathol 134:171-181

23. Kern B, Depner KR, Letz W, Rott M, Thalheim S, Nitschke B, Plagemann R, Liess B (1999) Incidence of classical swine fever (CSF) in wild boar in a densely populated area indicating CSF virus persistence as a mechanism for virus perpetuation. Zentralbl Veterinarmed B 46:63-67

24. Oesch B, Doherr M, Heim D, Fischer K, Egli S, Bolliger S, Biffiger K, Schaller O, Vandevelde M, Moser M (2000) Application of Prionics Western blotting procedure to screen for BSE in cattle regularly slaughtered at Swiss abattoirs. Arch Virol Suppl 189195
25. OIE (2004) Manual of diagnostic tests and vaccines for terrestrial animals, 5th edn. www.oie.int

26. Orge L, Simas JP, Fernandes AC, Ramos M, Galo A (2000) Similarity of the lesion profile of BSE in Portuguese cattle to that described in British cattle. Vet Rec 147:486-488

27. Schaller O, Fatzer R, Stack M, Clark J, Cooley W, Biffiger K, Egli S, Doherr M, Vandevelde M, Heim D, Oesch B, Moser M (1999) Validation of a western immunoblotting procedure for bovine $\operatorname{PrP}(\mathrm{Sc})$ detection and its use as a rapid surveillance method for the diagnosis of bovine spongiform encephalopathy (BSE). Acta Neuropathol 98:437-443

28. Seuberlich T, Botteron C, Wenker C, Cafe-Marcal VA, Oevermann A, Haase B, Leeb T, Heim D, Zurbriggen A (2006) Spongiform encephalopathy in a miniature zebu. Emerg Infect Dis 12:1950-1953

29. Simmons MM, Harris P, Jeffrey M, Meek SC, Blamire IW, Wells GA (1996) BSE in Great Britain: consistency of the neurohistopathological findings in two random annual samples of clinically suspect cases. Vet Rec 138:175-177

30. Sutmoller P, Casas OR (2002) Unapparent foot and mouth disease infection (sub-clinical infections and carriers): implications for control. Rev Sci Tech 21:519-529

31. Wells GA, Scott AC, Johnson CT, Gunning RF, Hancock RD, Jeffrey M, Dawson M, Bradley R (1987) A novel progressive spongiform encephalopathy in cattle. Vet Rec 121:419-420

32. Wells G, Hawkins S, Hadlow W, Spencer Y (1992) The discovery of bovine spongiform encephalopathy and observations on the vacuolar changes. In: Prusiner S, Collinge J, Powell J, Anderton B (eds) Prion diseases of humans and animals. Ellis Horwood, Chichester, pp 256-274

33. Wells GA, Wilesmith JW (1995) The neuropathology and epidemiology of bovine spongiform encephalopathy. Brain Pathol 5:91-103

34. Yamakawa Y, Hagiwara K, Nohtomi K, Nakamura Y, Nishijima M, Higuchi Y, Sato Y, Sata T (2003) Atypical proteinase K-resistant prion protein (PrPres) observed in an apparently healthy 23month-old Holstein steer. Jpn J Infect Dis 56:221-222 